\title{
Assessment of Global Cardiac Function in MSCT Imaging Using Fuzzy Connectedness Segmentation
}

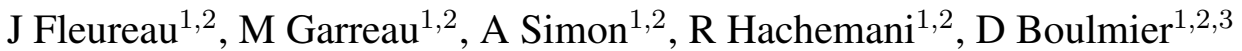 \\ ${ }^{1}$ INSERM, U642, Rennes, F-35000, France \\ ${ }^{2}$ Université de Rennes 1, LTSI, Rennes, F-35000, France \\ ${ }^{3} \mathrm{CHU}$ Rennes, Service de cardiologie et maladies vasculaires, Rennes, F-35000, France
}

\begin{abstract}
The goal of this work is to assess global cardiac function in terms of ventricular volume from Multi-Slice Computed Tomography dynamic dataset. We propose an approach for the segmentation of the left ventricle and the measurement of the ventricular volume along the whole cardiac cycle. It is based on the segmentation of the left cavities using a fuzzy connectedness algorithm. The interactive placement of a $3 D$ valvular plane is then used to cut through the segmented surface in order to extract the left ventricle. This method provides, with few interactions, the cardiac volume along the whole cardiac cycle and global parameters such as end-cardiac phases volumes and ejection fraction. Results have been compared to measures estimated during clinical routine with MSCT and MRI and have provided satisfying results. The specificity and the sensibility of the method have also been measured by a comparison with a manual segmentation of reference, leading to a very good specificity of the proposed approach.
\end{abstract}

\section{Introduction}

Cardiac function assessment from 3D image sequences has been greatly improved by the recent technical developments of 3D echography, cine-MRI and Multi-Slice Computed Tomography (MSCT). MSCT imaging offers significant advantages to study both cardiac anatomy and function, since it provides in a single exam, in a reduced acquisition time and with a high spatial resolution, 3D dynamic images of cardiac walls and cavities and of coronary vessels. Nevertheless, these dynamic 3D images lead to very large data sets from which the cardiac structures have to be extracted under strong constraints to face clinical requirements. This paper is focused on the segmentation of the left ventricular cavity for each 3D volume of the cardiac MSCT sequence, from which global 3D quantitative parameters are extracted to describe the left cardiac function. Several approaches have been proposed to solve seg- mentation problems in 3D imaging. Deformable models have been widely developed on 3D cardiac segmentation. To cite the main works, these models can be probabilistic models such as in [1,2] in cardiac MRI, or [3,4] in cardiac MSCT imaging. They can be also parametric models with variational approaches $[5,6]$ or implicit models with Level-Set methods [7] applied in cardiac MRI. Fuzzy Connectedness methods [8] and Graphcuts methods [9] have shown their interest for 3D segmentation in medical imaging, mainly applied in vascular and cerebral imaging, more recently in cardiac MRI [10]. Multi-agent approaches have also been considered for the analysis of brain images [11]. A multi-agent scheme combined with a supervised classification methodology to handle multi-object segmentation in cardiac MSCT imaging has recently been proposed in [12] The purpose of this paper is to measure the effectiveness of the fuzzy connectedness approach for the detection of the left cardiac cavity in MSCT dynamic images. The method which has been used is described, including the formation of a connectedness map, the definition of a valvular plane to separate left atrium and left ventricle and the extraction of global parameters. The obtained results are finally presented and discussed with a quantitative evaluation of the method.

\section{Methods}

The fuzzy connectedness (FC) approach provides a new formalization of the segmentation problem based on the graph theory. This approach seems to be adapted to the extraction of fuzzy anatomical shapes in medical imaging and presents capabilities for the detection of multi-objects. This paragraph resumes the basic aspects of this theory and describes how a simple version of $\mathrm{FC}$ has been adapted to be used in an efficient way on cardiac MSCT images.

A Fuzzy Connectedness algorithm. In classification problems considered in image segmentation, the initial idea proposed in [8] is to associate to each spel of an image a membership value significative of the strength of connectedness between this spel and the object of interest 
(OOI). These membership values can then be submitted to a final decision procedure. To initialize this process, the user needs to select one seed point (or more) belonging to the object to identify. The originality of the method lies in the definition of the connectedness concept.

If $V$ represents the set of spels of the image to segment (in 2D, a spel is equivalent to a pixel, in $3 \mathrm{D}$ it is a voxel), an affinity relation noted $\mu_{v}$ is firstly defined to compare two spels of the digital space $V . \mu_{v}$ is given by:

$$
\begin{aligned}
\mu_{v}: V \times V & \longrightarrow \\
(c, d) & \longmapsto \mu_{v}(c, d)
\end{aligned}
$$

and it defines the similarity between two spels $(c, d)$. This relationship can be defined according to several criteria often combined, including in most cases [8] adjacency and homogeneity properties. The definition of $\mu_{v}$ is here composed of two terms:

$$
\mu_{v}(c, d)=\mu_{a d}(c, d) \mu_{h}(c, d)
$$

The first term is a spatial similarity term, reduced to a 6connexity relationship to select the spatial candidate spels $(d)$, neighbours of the spel $(c)$. The second term is an homogeneity term brought by the image information for each spel and is defined by:

$$
\mu_{h}(c, d)=\mu_{h}^{1}(c, d) \mu_{h}^{2}(c, d)
$$

which is composed of: (1) a local affinity term defined by:

$$
\mu_{h}^{1}(c, d)=\frac{1}{1+|f(c)-f(d)|}
$$

where the function $f$ is linked to the spel local density in the images; (2) a global affinity term defined by:

$\mu_{h}^{2}(c, d)=\left\{\begin{array}{c}\frac{1}{1+|f(c)-f(o)|}, \text { if } f(o) \text { belongs to the mode } \\ \text { defined for the object of interest; } \\ 0 \text { otherwise. }\end{array}\right.$

where $o$ is the seed point initially chosen by the user in the object of interest.

One of the main point of Fuzzy Connectedness algorithms stays in the construction of a set of paths $\mathbb{P}$. Each path $p_{c d} \in \mathbb{P}$ linking two spels $c$ and $d$ is a finite serie of spatially connected spels starting with the spel $c$ and ending with the spel $d$ :

$$
\begin{gathered}
p_{c d}=\left(s^{(0)}, s^{(1)}, \ldots, s^{(i)}, \ldots, s^{(n-1)}, s^{(n)}\right) \\
\text { with } s^{(0)}=c \text { and } s^{(n)}=d \text { where } s^{(i)} \in V, \forall i
\end{gathered}
$$

The informations of continuity and connectedness introduced by these paths provide a global point of view to decide of the association of one spel to an object. They give also a mean to face with local noise and artefact that can be present in the images.
According to the FC theory, a strength is associated to each path by introducing a function $\mu_{\mathbb{N}}$ called Membership Function, defined as follows:

$$
\begin{gathered}
\mu_{\mathbb{N}}\left(p_{c d}\right)=\min \left[\mu_{v}\left(s^{(0)}, s^{(1)}\right), \mu_{v}\left(s^{(1)}, s^{(2)}\right),\right. \\
\left.\quad \ldots, \mu_{v}\left(s^{(i)}, s^{(i+1)}\right), \ldots, \mu_{v}\left(s^{(n-1)}, s^{(n)}\right)\right]
\end{gathered}
$$

and the strength of the strongest path linking two any spels $c$ and $d$ is given by the definition of the Fuzzy Connectedness $\mu_{\mathbb{K}}(c, d)$ which is:

$$
\mu_{\mathbb{K}}(c, d)=\max _{p_{c d} \in \mathbb{P}}\left[\mu_{\mathbb{N}}\left(p_{c d}\right)\right]
$$

This "Max-Min" approach is the main point of the FC theory. To segment an object, it is necessary to select a seed point inside the OOI, and a Connectedness map is therefore provided by computing the connectedness between each spel of the digital space and this seed point. Finally, the user has to threshold this map according to the object of interest. Complementary versions of FC have been also proposed, in particular the Relative Fuzzy Connectedness approach (RFC) [13] to avoid the thresholding step by the use of two seed points (one in the background and one in the OOI).

Connectedness Map and Dijkstra Algorithm. However, computing such a map requires the evaluation of numerous "Max-Min" which is quite time consuming. Powerful algorithms can be used to solve such problem. Dijkstra algorithm has been retained in this study and allows to find the shortest path in a graph under the constraint of a specific cost. The problem to solve here is effectively a problem of graph searching where the nodes are the spels, the links are the paths between two spels and the cost, the affinity function. Considering this Dijkstra algorithm, the construction of the connectedness map has been realized with low costs in terms of memory use and rapidity. This algorithm begins from a central seed point in the OOI and expands the research in the graph in a width way.

Extension in time and extraction of clinical parameters. In order to study the global cardiac function, the connectedness segmentation algorithm is applied on dynamic MSCT datasets. Considering that the left cavity is not submitted to a large global displacement, the same seed point can be used for all the 3D volumes of one patient database, provided that this point is positioned about the centre of the cavity. From this seed point, the left cavities are segmented along the whole cardiac cycle. As illustrated by the figure 1(a), even with a low threshold, it generally results to the extraction of the endocardium, but also of the beginning of the aorta and of part of the left auricle. Depending on the contrast between left and right cavities, part of the right ventricle may also be extracted (cf. figure 1(b)).

Therefore, because segmented structures are not limited to the left ventricle, disjoining it from the other structures 


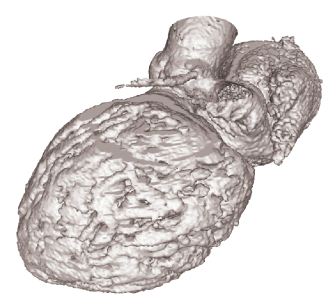

(a)

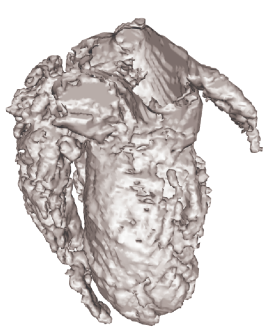

(b)
Figure 1. Segmentation result: (a) left cavities represented according to the right anterior oblique view; (b) part of the right ventricle may also be extracted.

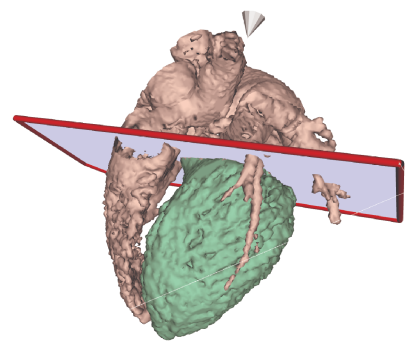

Figure 2. 3D plane used to delimit the left ventricle.

is needed. This is realized by the interactive placement of a 3D-plane to cut throught the reconstructed surface at the position of the valvular plane (cf. figure 2). Because the base of the ventricle is globally submitted to translational displacement towards the apex, this placement has to be adjusted, from time to time along the cardiac cycle. From the position of this plane, the largest connected region is extracted, corresponding to the left ventricle. The left ventricle volume and the ejection fraction are then computed according to the acquisition resolution.

\section{Results}

The approach has been applied to MSCT dynamic datasets acquired within the IMOP project (IMaging for the Optimization of biventricular Pacing) which goal is to define an optimization method for Cardiac Resynchronization Therapy. Five patients have been considered (adults with congestive heart failure (dyssynchrony)). Datasets have been acquired with a General Electric 64 slices MSCT with contrast agent injection and retrospective cardiac synchronization. Acquired data consists in 19 or 20 $3 \mathrm{D}$ volumes representing a whole cardiac cycle. The figure 3 represents two examples of ventricular volume computed along the whole cardiac cycle.

For the considered patients, two other volume measurements have been provided: routine end-diastolic and endsystolic volumes computed from MRI and from MSCT ac-

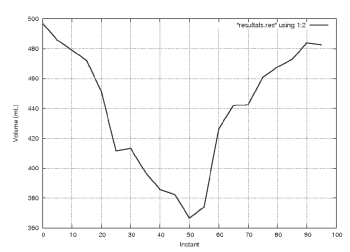

(a)

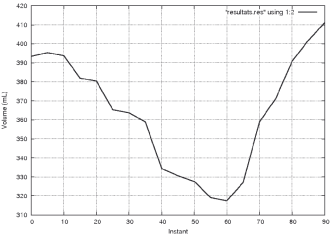

(b)
Figure 3. Left ventricle volume along the cardiac cycle for two different patients.

\begin{tabular}{|c|c|c|c|c|c|c|c|}
\hline \multirow{2}{*}{\multicolumn{2}{|c|}{ routine MSCT }} & \multirow{2}{*}{\multicolumn{2}{|c|}{ routine MRI }} & \multicolumn{4}{|c|}{ MSCT: proposed method } \\
\hline & & & & \multicolumn{2}{|c|}{$\begin{array}{c}\text { considered } \\
\text { phases }\end{array}$} & \multicolumn{2}{|c|}{$\begin{array}{c}\text { absolute } \\
\text { values }\end{array}$} \\
\hline EDV & ESV & EDV & ESV & EDV & ESV & EDV & ESV \\
\hline 388 & 318 & 373 & 343 & 365 & 309 & 368 & 294 \\
\hline 320 & 267 & 293 & 263 & 326 & 243 & 330 & 243 \\
\hline n.a. & n.a. & 184 & 140 & n.a. & n.a. & 176 & 117 \\
\hline
\end{tabular}

Figure 4. End diastolic volume (EDV) and end-systolic volume (ESV) for three patients: clinical routine with MSCT, clinical routine with MRI and proposed method method with MSCT (two measures: at the phases used for routine measures with MSCT and absolute (minimal and maximal) values). (n.a: not available)

quisitions on the clinical stations. It is important to notice that these routine MSCT and MRI volume measurements are made by the clinician only at the visually estimated end-diastolic and end-systolic phases. All these values can be compared on figure 4 for three cases.

Considering the same phases, differences between routine MSCT measurements and the proposed approach are less than $6 \%$. This is explained by the fact that, by opposition to the clinical measurements, the proposed approach does not include papillary muscles in the left ventricle.

Considering the absolute minimal and maximal volumes along the cardiac cycle, the difference with routine MSCT raise up to $9 \%$ and with routine MRI up to $16 \%$. So, the automatic selection of the end-diastolic and endsystolic phases increase the differences with clinical measurements. It highlights the fact that the manually selected phase (usually realized by the dynamic visualization of one slice) does not necessarily results to the selection of the phases with minimal or maximal volumes.

To evaluate in a more precise way the quality of the segmentation, a comparison with a segmentation of reference has been realized. On five MSCT volumes (one volume for one patient), in ten slices selected from the basal to the apical part of the heart, one expert has validated the manual delineation of the endocardium. The results of the 3D segmentation obtained by the RFC method (with two seed points, in the atrium and in the left cavity, avoiding the selection of the valvular plane) have been compared to the manual segmentation in each of the ten slices. The sensibil- 


\begin{tabular}{|c|c|c|c|}
\hline Patient & SE & SP & E \\
\hline 1 & 67.90 & 99.85 & 3.62 \\
\hline 2 & 49.28 & 99.95 & 6.20 \\
\hline 3 & 32.56 & 99.64 & 6.93 \\
\hline 4 & 65.88 & 98.74 & 3.66 \\
\hline 5 & 65.88 & 98.74 & 3.61 \\
\hline
\end{tabular}

Figure 5. Sensibility (SE), specificity (SP) of the method and global error (E) computed for five volumes by considering the mean value on ten slices per volume.

ity and the specificity of the method have been estimated with the computation of the true and false positive points number, the true and false negative points number, by considering the voxels as the points. The results obtained for each database and by considering the mean value of measures for the ten slices, are given in figure 5 .

The mean error computed for the set of databases is low, it remains about $5 \%$ with an error between $3.5 \%$ and $7 \%$. These results show the good behaviour of the method with computing time acceptable in clinical use. In particular, the method has a good specificity (a mean value of 99\%), which shows a low over-detection. The sensibility of the method is widely inferior, with a mean value of $56.3 \%$ which is mainly due to the difficulty for the clinician to delimitate precisely, in some parts of the images, the endocardium without papillary muscles.

\section{Discussion and conclusions}

In this work, a segmentation method based on fuzzy connectedness is proposed to assess global cardiac function in MSCT dynamic imaging. From one unique seed point, the segmentation process, based on a connectedness map computed with the Dijkstra algorithm, extracts left cavities during the whole cardiac cycle. A plane is then used to cut through the resulting surface in order to extract the left endocardium. The comparison of computed left ventricular volume to clinical measurements (with MSCT and MRI), such as the comparison of the segmentation with an expert segmentation of reference, have provided satisfying results. Future works will deal with the extension of the quantitative evaluation procedure to more patients and to a comparison to other segmentation methods.

\section{Acknowledgements}

This work is supported by the French Research Minister $\left(\mathrm{n}^{\mathrm{o}} 04 \mathrm{~T} 187-188-189-190 \mathrm{CITH}\right)$ in IMOP project (IMaging for the Optimization of biventricular Pacing).

\section{References}

[1] Lorenzo-Valdes M, Sanchez-Ortiz G, Elkinton A, Mohiaddin R, Rueckert D. Segmentation of 4D cardiac MR im- ages using a probabilistic atlas and the EM algorithm. Med Image Anal 2004;8(3):255-265.

[2] Frangi A, Rueckert D, Schnabel J, Niessen W. Automatic construction of multiple-object three-dimensional statistical shape models: application to cardiac modeling. IEEE Trans Med Imag 2006;21(9):1151-1166.

[3] Ecabert O, Peters J, Schramm H, Lorenz C, von Berg J, Walker M, Vembar M, Olszewski M, Subramanyan K, Lavi G, Weese J. Automatic model-based segmentation of the heart in CT images. IEEE Trans Med Imag 2008; 27(9):1189-1201.

[4] Garreau M, Simon A, Boulmier D, Coatrieux J, Le Breton H. Assessment of left ventricular function in cardiac MSCT imaging by a $4 \mathrm{D}$ hierarchical surface-volume matching process. International Journal of Biomedical Imaging 2006;ID 37607:1-10.

[5] McInerney T, Terzopoulos D. Deformable models in medical image analysis : A survey. Med Image Anal 1996; 1(2):91-108.

[6] Montagnat J, Delingette H. 4D deformable models with temporal constraints: application to 4D cardiac image segmentation. Med Image Anal 2005;9(1):87-100.

[7] Paragios N. A level set approach for shape-driven segmentation and tracking of the left ventricle. IEEE Trans Med Imag 2006;22(6):773-776.

[8] Udupa J, Samarasekera S. Fuzzy connectedness and object definition: Theory, algorithms, and applications in image segmentation. Graphical Models and Image Processing 1996;58(3):246-261.

[9] Boykov Y, Kolmogorov V. An experimental comparison of min-cut/max- flow algorithms for energy minimization in vision. IEEE Trans Pat Anal Mach Intel 2004;26(9):1124 1137.

[10] Pednekar A, Kakadiaris I. Image segmentation based on fuzzy connectedness using dynamic weights. IEEE Transactions on Image Processing 2006;15(6):1555-1562.

[11] Richard N, Dojat M, Garbay C. Automated segmentation of human brain MR images using a multi-agent approach. Artificial Intelligence in Medicine 2004;30(2):153-176.

[12] Fleureau J, Garreau M, Boulmier D, Hernandez A. 3D multi-object segmentation of cardiac MSCT imaging by using a multi-agent approach. Proceedings IEEE EMBS 2007;6003-6006.

[13] Udupa J, Saha P, Lotufo R. Relative fuzzy connectedness and object definition: Theory, algorithms, and applications in image segmentation. IEEE Trans Pat Anal Mach Intel 2002;24(11):1485-1492.

Address for correspondence:

Mireille GARREAU

LTSI, Campus de Beaulieu, Université de Rennes 1, 263 Avenue du General Leclerc - CS 74205 - 35042 Rennes Cedex, France. mireille.garreau@univ-rennes1.fr 